\title{
A Nonlinear Schrödinger Equation Resonating at an Essential Spectrum
}

\author{
Shaowei Chen and Haijun Zhou \\ School of Mathematical Sciences, Huaqiao University, Quanzhou 362021, China \\ Correspondence should be addressed to Shaowei Chen; swchen6@163.com \\ Received 12 December 2015; Accepted 14 February 2016 \\ Academic Editor: Jacopo Bellazzini
}

Copyright (c) 2016 S. Chen and H. Zhou. This is an open access article distributed under the Creative Commons Attribution License, which permits unrestricted use, distribution, and reproduction in any medium, provided the original work is properly cited.

We consider the nonlinear Schrödinger equation $-\Delta u+f(u)=V(x) u$ in $\mathbb{R}^{N}$. The potential function $V$ satisfies that the essential spectrum of the Schrödinger operator $-\Delta-V$ is $[0,+\infty)$ and this Schrödinger operator has infinitely many negative eigenvalues accumulating at zero. The nonlinearity $f$ satisfies the resonance type condition $\lim _{|t| \rightarrow \infty} f(t) / t=0$. Under some additional conditions on $V$ and $f$, we prove that this equation has infinitely many solutions.

\section{Introduction and Statement of Results}

In this paper, the following nonlinear Schrödinger problem in $\mathbb{R}^{N}(N \geq 3)$ is considered:

$$
-\Delta u=V(x) u+g(u) \quad \text { in } \mathbb{R}^{N} .
$$

The nonlinearity $g$ satisfies the resonance type condition:

$$
\lim _{|t| \rightarrow \infty} \frac{g(t)}{t} \in \sigma_{\text {ess }}(T) .
$$

Here, $\sigma_{\text {ess }}(T)$ denotes the essential spectrum (see [1, Chapter 7] or [2, Chapter 7.4]) of the Schrödinger operator $T$ defined as follows:

$$
\begin{aligned}
T: L^{2}\left(\mathbb{R}^{N}\right) & \longrightarrow L^{2}\left(\mathbb{R}^{N}\right), \\
u & \longmapsto-\Delta u-V u,
\end{aligned}
$$

with domain $D(T):=\left\{u \in L^{2}\left(\mathbb{R}^{N}\right) \mid T u \in L^{2}\left(\mathbb{R}^{N}\right)\right\}$.

Equation (1) arises in quantum mechanics and is related to the study of the nonlinear Schrödinger equation for a particle in an electromagnetic field and it has attracted considerable attention from researchers in recent decades. One can see [3-6] and the references therein. However, there are very few results on (1) with the resonance type condition (2).
The following nonlinear elliptic problem with resonance type conditions in bounded domain has been studied by many authors and numerous existence and multiplicity results have been obtained in the past forty years:

$$
-\Delta u=h(x, u) \quad \text { in } \Omega, u=0 \text { on } \partial \Omega .
$$

Here, $\Omega$ is a bounded domain in $\mathbb{R}^{N}$, and $g$ satisfies the resonance type condition:

$$
\lim _{|t| \rightarrow \infty} \frac{h(x, t)}{t}=v_{k}
$$

$v_{k}$ is the $k$ th eigenvalue of $-\Delta$ with 0 -Dirichlet boundary condition on $\partial \Omega$. We refer to [7-10] and references therein for more detailed discussions of some historical results. This problem has deeply inspired developments in critical point theory in the last forty years, such as the Landesman-Lazer type conditions (e.g., $[8,10])$, Morse theory (e.g., [9]), and the variational reduction method (e.g., [11]). The difficulty of this problem lies in the proof of the boundedness of the Palais-Smale sequence or the $(C)_{c}$ sequence (see Definition 5 in Section 2) of its corresponding functional.

The resonant Schrödinger problem (1) is much more difficult than the bounded domain case and it has fewer studies. Unlike the case of bounded domains for which the linear operators $-\Delta$ in bounded domain are compact, there are continuous spectra of the linear operator $T$. Moreover, the 
proofs of boundedness and compactness of the $(C)_{c}$ sequence of the corresponding functional of (4) are greatly different from and more difficult than the case of bounded domain (see $[4,5,12])$.

More precisely, this paper considers the nonlinear Schrödinger equation:

$$
-\Delta u+f(u)=V(x) u, \quad u \in H^{1}\left(\mathbb{R}^{N}\right) .
$$

Here, $N \geq 3$ and $H^{1}\left(\mathbb{R}^{N}\right)$ is the Sobolev space:

$$
\begin{aligned}
& H^{1}\left(\mathbb{R}^{N}\right):=\left\{u:\left.\mathbb{R}^{N} \longrightarrow \mathbb{R}\left|\int_{\mathbb{R}^{N}}\right| \nabla u\right|^{2} d x\right. \\
& \left.\quad+\int_{\mathbb{R}^{N}} u^{2} d x<+\infty\right\} .
\end{aligned}
$$

For $V: \mathbb{R}^{N} \rightarrow \mathbb{R}$, the following is assumed:

$\left(\mathbf{V}_{1}\right)$ Consider $V \in C^{1}\left(\mathbb{R}^{N} \backslash\{0\}\right), V \geq 0$ in $\mathbb{R}^{N} \backslash$ $\{0\}, \lim _{|x| \rightarrow \infty} V(x)=0, V \in L_{\text {loc }}^{s}\left(\mathbb{R}^{N}\right)$ with $s=$ $\max \{2, N / 2\}$ if $N \geq 3$ and $N \neq 4, V \in L_{\text {loc }}^{s}\left(\mathbb{R}^{N}\right)$ for some $s>2$ if $N=4$, and there exist $R>0,0<\beta<2$, and $C>0$ such that, for $|x| \geq R$,

$$
V(x) \geq C|x|^{-\beta} \text {. }
$$

$\left(\mathbf{V}_{2}\right)$ For any $x \in \mathbb{R}^{N} \backslash\{0\}, x \cdot \nabla V(x) \leq 0$ and there exists $0<\gamma<2$ such that

$$
\gamma V(x)+x \cdot \nabla V(x) \geq 0, \quad \forall x \in \mathbb{R}^{N} \backslash\{0\} .
$$

For $f$, the following is assumed:

$\left(\mathbf{f}_{1}\right) f: \mathbb{R} \rightarrow \mathbb{R}$ is a continuous function:

$$
\begin{aligned}
a_{0} & :=\lim _{t \rightarrow 0} \frac{f(t)}{t}>0, \\
\lim _{|t| \rightarrow \infty} \frac{f(t)}{t} & =0, \\
t f(t) & \geq 0, \quad \forall t \in \mathbb{R} .
\end{aligned}
$$

$\left(\mathbf{f}_{2}\right)$ There exists $D>0$ such that, for any $t \in \mathbb{R}$,

$$
F(t) \leq D t f(t)
$$

Here,

$$
F(t)=\int_{0}^{t} f(s) d s, \quad t \in \mathbb{R} .
$$

For $t \in \mathbb{R}$, let

$$
\begin{aligned}
& g(t)=a_{0} t-f(t), \\
& \widetilde{F}(t)=F(t)-\frac{1}{2} t f(t) .
\end{aligned}
$$

$\left(\mathbf{f}_{3}\right)$ For any $t \in \mathbb{R}, \widetilde{F}(t) \geq 0$ and there exists $\delta \in\left(0, a_{0}\right)$ such that if $|g(t)| \geq\left(a_{0}-\delta\right)|t|$, then

$$
\widetilde{F}(t) \geq \delta \text {. }
$$

$\left(\mathbf{f}_{4}\right)$ For any $t \in \mathbb{R}, f(-t)=-f(t)$.

The main result of this paper is as follows.

Theorem 1. Suppose that $\left(\mathbf{V}_{\mathbf{1}}\right)-\left(\mathbf{V}_{\mathbf{2}}\right)$ and $\left(\mathbf{f}_{\mathbf{1}}\right)-\left(\mathbf{f}_{\mathbf{4}}\right)$ are satisfied; then, problem (6) has infinitely many solutions.

Remark 2. (i) Under the assumptions $\left(\mathbf{V}_{\mathbf{1}}\right)$ and $\left(\mathbf{V}_{\mathbf{2}}\right)$, the essential spectrum of $T$ defined by (3) is $[0,+\infty$ ) (see Lemma 3 in Section 2). Therefore, condition (11) indicates that $f$ satisfies a resonance type condition at the essential spectrum of $T$.

(ii) A typical example for $V$ which satisfies $\left(\mathbf{V}_{\mathbf{1}}\right)-\left(\mathbf{V}_{\mathbf{2}}\right)$ is

$$
V(r, \theta)=\frac{P(\theta)}{r^{\beta}} .
$$

Here, $r=|x|, \theta=x /|x| \in S^{N-1}:=\left\{y \in \mathbb{R}^{N}|| y \mid=1\right\}$, $0<\beta<\min \{2, N / 2\}, P \in C^{1}\left(S^{N-1}\right)$, and $P>0$ in $S^{N-1}$.

(iii) There are many functions satisfying $\left(\mathbf{f}_{\mathbf{1}}\right)-\left(\mathbf{f}_{\mathbf{4}}\right)$. Let $G(t)=\int_{0}^{t} g(s) d s$. Using

$$
\frac{d}{d t}\left(\frac{G(t)}{t^{2}}\right)=\frac{2 \widetilde{F}(t)}{t^{3}},
$$

we can construct a function $f$ satisfying $\left(\mathbf{f}_{\mathbf{1}}\right)-\left(\mathbf{f}_{\mathbf{4}}\right)$ from a given $\widetilde{F}$. For example, let

$$
\widetilde{F}(t)=\frac{\sigma|t|^{3}}{1+|t|^{5 / 2}}, \quad t \in \mathbb{R},
$$

where

$$
\sigma=\frac{a_{0}}{4 \tau}, \quad \tau=\int_{0}^{+\infty} \frac{d s}{1+|s|^{5 / 2}} .
$$

Then, by (19), for $t \geq 0$,

$$
G(t)=2 \sigma t^{2} \int_{0}^{t} \frac{d s}{1+|s|^{5 / 2}}
$$

and, for $t<0, G(t)=G(-t)$. It follows that

$$
\begin{aligned}
& g(t)=4 \sigma t \int_{0}^{t} \frac{d s}{1+|s|^{5 / 2}}+\frac{2 \sigma t^{2}}{1+|t|^{5 / 2}} \quad \text { if } t \geq 0, \\
& g(t)=-g(-t) \quad \text { if } t<0,
\end{aligned}
$$

and $f(t)=a_{0} t-g(t)$ for any $t \in \mathbb{R}$. Because the even function $g(t) / t$ is strictly increasing in $(0,+\infty), g(t) / t \rightarrow a_{0}$ as $t \rightarrow+\infty$, it can be deduced that $f$ satisfies $t f(t)>0$, $\forall t \in \mathbb{R} \backslash\{0\}$. Moreover, it is easy to verify that $f(t)=a_{0} t+o(t)$ as $t \rightarrow 0, f(t)=(2 / 3) \sigma t^{-1 / 2}+o\left(t^{-1 / 2}\right)$ as $|t| \rightarrow \infty, F(t)=$ $(1 / 2) a_{0} t^{2}+o\left(t^{2}\right)$ as $t \rightarrow 0$, and $F(t)=(4 / 3) \sigma|t|^{1 / 2}+o\left(|t|^{1 / 2}\right)$ as $|t| \rightarrow \infty$. Because the even function $g(t) / t$ is strictly increasing in $(0,+\infty), g(t) / t \rightarrow a_{0}$ as $t \rightarrow+\infty$, and $\widetilde{F}(t) \rightarrow$ $+\infty$ as $|t| \rightarrow+\infty$, it can be deduced that, for sufficiently small $\delta>0$, if $|g(t)| /|t| \geq a_{0}-\delta$, then $\widetilde{F}(t) \geq \delta$. Therefore, $f$ satisfies $\left(\mathbf{f}_{1}\right)-\left(\mathbf{f}_{4}\right)$. 
Let

$$
X=H^{1}\left(\mathbb{R}^{N}\right)
$$

The inner product and the norm in $X$ are

$$
(u, v)=\int_{\mathbb{R}^{N}}\left(\nabla u \nabla v+a_{0} u v\right) d x, \quad\|u\|=(u, u)^{1 / 2}
$$

respectively. Here, $a_{0}$ is the constant in (10).

Let

$$
\begin{aligned}
\Phi(u)= & \frac{1}{2} \int_{\mathbb{R}^{N}}|\nabla u|^{2} d x-\frac{1}{2} \int_{\mathbb{R}^{N}} V(x) u^{2} d x \\
& +\int_{\mathbb{R}^{N}} F(u) d x, \quad u \in X .
\end{aligned}
$$

Then, for any $u, v \in X$, the Gateaux derivative of $\Phi$ is as follows:

$$
\begin{aligned}
\left\langle\Phi^{\prime}(u), v\right\rangle= & \int_{\mathbb{R}^{N}} \nabla u \nabla v d x-\int_{\mathbb{R}^{N}} V(x) u v d x \\
& +\int_{\mathbb{R}^{N}} f(u) v d x .
\end{aligned}
$$

It follows that $u \in X$ is a solution of (6) if and only if $u$ is a critical point of $\Phi$. In Section 2, it is shown that $\Phi$ satisfies the Cerami condition. In Section 3, an abstract critical point theorem from [13] is used to show that $\Phi$ has infinitely many critical points.

Notation. Let $E$ be Banach space. We denote the dual space of $E$ by $E^{\prime}$ and denote strong and weak convergence in $E$ by $\rightarrow$ and $\rightarrow$, respectively. For $\varphi \in C^{1}(E, \mathbb{R})$, we denote the Fréchet derivative of $\varphi$ at $u$ by $\varphi^{\prime}(u)$. The Gateaux derivative of $\varphi$ is denoted by $\left\langle\varphi^{\prime}(u), v\right\rangle, \forall u, v \in E . L^{p}\left(\mathbb{R}^{N}\right)$ denotes the standard $L^{p}$ space $(1 \leq p \leq \infty)$. We use $O(h)$ and $o(h)$ to mean $|O(h)| \leq C|h|$ and $o(h) /|h| \rightarrow 0$, respectively.

\section{The Cerami Condition for $\Phi$}

Lemma 3. If $V$ satisfies the assumptions $\left(\mathbf{V}_{\mathbf{1}}\right)$, then the essential spectrum of the Schrödinger operator $T$ defined by (3) is $[0,+\infty)$ and $T$ has infinitely many negative eigenvalues accumulating at zero.

Proof. Because $V$ satisfies $\left(\mathbf{V}_{1}\right)$, by [14, Theorem XIII.15(b)] or [2, Theorem 10.29(b)], the essential spectrum of the Schrödinger operator $T$ defined by $(3)$ is $[0,+\infty)$. The second result of this lemma comes from [2, Theorem 10.31] or [14, Theorem XIII.6(a)].

Lemma 4. If $V$ satisfies the assumptions $\left(\mathbf{V}_{\mathbf{1}}\right)$ and $\left(\mathbf{V}_{\mathbf{2}}\right)$, then $T$ has no eigenvalue in $[0,+\infty)$.

Proof. Because $V$ satisfies the assumptions $\left(\mathbf{V}_{\mathbf{1}}\right)$ and $\left(\mathbf{V}_{\mathbf{2}}\right)$, by $[15$, Corollary 2], to prove this lemma, it suffices to prove that
$V$ satisfies the conditions I and II in [15, Corollary 2]. For this, it suffices to prove that, for $0<\alpha<2$, the functions

$$
\begin{aligned}
& M(x)=\int_{|x-y| \leq 1}|V(y)| \cdot|x-y|^{4-N-\alpha} d y, \\
& L(x)=\int_{|x-y| \leq 1}|y \nabla V(y)| \cdot|x-y|^{4-N-\alpha} d y
\end{aligned}
$$

are bounded functions in $\mathbb{R}^{N}$. Because $\lim _{|x| \rightarrow \infty} V(x)=0$ and $0<\alpha<2$, there exists $R^{\prime}>0$ such that, for $|x|>R^{\prime}$,

$$
\begin{aligned}
M(x) & \leq \int_{|x-y| \leq 1}|x-y|^{4-N-\alpha}=\int_{|y| \leq 1}|y|^{4-N-\alpha} d y \\
& <+\infty
\end{aligned}
$$

Because $V \in L_{\text {loc }}^{N / 2}\left(\mathbb{R}^{N}\right)$, for $|x| \leq R^{\prime}$, the following is true:

$$
\begin{gathered}
M(x) \leq\left(\int_{|x-y| \leq 1}|V(y)|^{N / 2} d y\right)^{2 / N} \\
\cdot\left(\int_{|x-y| \leq 1}|x-y|^{N(4-N-\alpha) /(N-2)}\right)^{(N-2) / N} \\
=\left(\int_{|y| \leq R^{\prime}+1}|V(y)|^{N / 2} d y\right)^{2 / N} \\
\cdot\left(\int_{|y| \leq 1}|y|^{N(4-N-\alpha) /(N-2)}\right)^{(N-2) / N} .
\end{gathered}
$$

Because $0<\alpha<2$, the following holds:

$$
\int_{|y| \leq 1}|y|^{N(4-N-\alpha) /(N-2)}<+\infty .
$$

Combining (29) and (30) yields that $M$ is a bounded function in $\mathbb{R}^{N}$. From $\left(\mathbf{V}_{2}\right)$, we have $L(x) \leq \gamma M(x), \forall x \in \mathbb{R}^{N}$. It follows that $L$ is also a bounded function in $\mathbb{R}^{N}$.

Definition 5 (see [16]). Let $E$ be a Banach space. Let $J \in$ $C^{1}(E, \mathbb{R})$ and $c \in \mathbb{R}$. One can call the fact that $J$ satisfies the Cerami condition at $c$, denoted by $(C)_{c}$ condition, if for any $\left\{u_{n}\right\} \subset E$ satisfying

$$
\begin{aligned}
\lim _{n \rightarrow \infty} J\left(u_{n}\right) & =c, \\
\lim _{n \rightarrow \infty}\left(1+\left\|u_{n}\right\|\right)\left\|J^{\prime}\left(u_{n}\right)\right\| & =0,
\end{aligned}
$$

there exists $u \in E$ such that, up to a subsequence, $\left\|u_{n}-u\right\| \rightarrow$ 0 as $n \rightarrow \infty$. If $J$ satisfies the $(C)_{c}$ condition for every $c \in$ $\mathbb{R}$, then $J$ is said to satisfy the Cerami condition. A sequence $\left\{u_{n}\right\} \subset E$ satisfying (32) is called a $(C)_{c}$ sequence of $J$.

It was shown in [13] that the Cerami condition actually suffices to get a deformation theorem (see [8]) and then, by standard minimax arguments, it allows rather general minimax results. 
Lemma 6. Suppose that $V$ satisfies that $V \in L_{l o c}^{N / 2}\left(\mathbb{R}^{N}\right)$ and $\lim _{|x| \rightarrow \infty} V(x)=0$. If $u_{n} \rightarrow u$ in $X$, then

$$
\lim _{n \rightarrow \infty} \int_{\mathbb{R}^{N}} V(x) u_{n}^{2} d x=\int_{\mathbb{R}^{N}} V(x) u^{2} d x .
$$

Proof. Because $u_{n} \rightarrow u$ in $X,\left\{\left\|u_{n}\right\|\right\}$ is bounded. It follows that

$$
M:=\sup _{n} \int_{\mathbb{R}^{N}}\left|u_{n}-u\right|^{2} d x<+\infty .
$$

From $\lim _{|x| \rightarrow \infty} V(x)=0$, we deduce that, for any $\epsilon>0$, there exists $R_{\epsilon}>0$ such that

$$
\sup _{|x| \geq R_{\varepsilon}}|V(x)|<\frac{\epsilon}{M} .
$$

It follows that

$$
\begin{aligned}
& \int_{|x| \geq R_{\epsilon}}|V(x)| \cdot\left|u_{n}-u\right|^{2} d x \\
& \leq \sup _{|x| \geq R_{\epsilon}}|V(x)| \cdot \int_{\mathbb{R}^{N}}\left|u_{n}-u\right|^{2} d x<\epsilon .
\end{aligned}
$$

For $y \in \mathbb{R}^{N}$ and $r>0$, let

$$
B_{\mathbb{R}^{N}}(y, r)=\left\{x \in \mathbb{R}^{N}|| x-y \mid<r\right\} .
$$

Because $V \in L^{N / 2}\left(B_{\mathbb{R}^{N}}\left(0, R_{\epsilon}\right)\right)$ and $u_{n} \rightarrow u$ in $X$, by Lemma 2.13 of [6], we have

$$
\lim _{n \rightarrow \infty} \int_{|x|<R_{\epsilon}} V(x) u_{n}^{2} d x=\int_{|x|<R_{\varepsilon}} V(x) u^{2} d x .
$$

Combining (36) and (38) yields (33).

Lemma 7. Let $\left\{u_{n}\right\} \subset X$ be a $(C)_{c}$ sequence of $\Phi$. Then, up to a subsequence, $\left\{\left\|u_{n}\right\|\right\}$ is bounded.

Proof. By Definition 5, we have

$$
\begin{aligned}
\lim _{n \rightarrow \infty} \Phi\left(u_{n}\right) & =c, \\
\lim _{n \rightarrow \infty}\left(1+\left\|u_{n}\right\|\right)\left\|\Phi^{\prime}\left(u_{n}\right)\right\| & =0 .
\end{aligned}
$$

Arguing indirectly, assume that $\left\|u_{n}\right\| \rightarrow \infty$ and set $w_{n}=$ $u_{n} /\left\|u_{n}\right\|$.

Case 1. $\left\{w_{n}\right\}$ is nonvanishing; that is, there exist $y_{n} \in \mathbb{R}^{N}$ and $\alpha>0$ such that

$$
\int_{B_{\mathbb{R}^{N}}\left(y_{n}, 1\right)} w_{n}^{2} d x \geq \alpha, \quad n=1,2, \ldots
$$

Set $\widetilde{w}_{n}=w_{n}\left(\cdot+y_{n}\right)$. Then, by (40), $\widetilde{w}_{n} \rightarrow w \neq 0$ in $X$. $\infty$,

By (39) and (27), for any $\phi \in C_{0}^{\infty}\left(\mathbb{R}^{N}\right)$, we have, as $n \rightarrow$

$$
\begin{aligned}
& \int_{\mathbb{R}^{N}} \nabla u_{n} \nabla \phi d x-\int_{\mathbb{R}^{N}} V(x) u_{n} \phi d x+\int_{\mathbb{R}^{N}} f\left(u_{n}\right) \phi d x \\
& \quad=o(1) .
\end{aligned}
$$

It follows that, as $n \rightarrow \infty$,

$$
\begin{gathered}
\int_{\mathbb{R}^{N}} \nabla \widetilde{w}_{n} \nabla \phi d x-\int_{\mathbb{R}^{N}} V\left(x+y_{n}\right) \widetilde{w}_{n} \phi d x \\
+\int_{\mathbb{R}^{N}} \frac{f\left(\widetilde{u}_{n}\right)}{\widetilde{u}_{n}} \widetilde{w}_{n} \phi d x=o(1),
\end{gathered}
$$

where $\tilde{u}_{n}=u_{n}\left(\cdot+y_{n}\right)$. If $\lim \left|y_{n}\right|=+\infty$, sending $n \rightarrow \infty$ in (42), by (11), $\lim _{|x| \rightarrow \infty} V(x)=0$ and the fact that, for $x \in\{x \in$ $\left.\mathbb{R}^{N} \mid w \neq 0\right\},\left|\widetilde{u}_{n}(x)\right| \rightarrow+\infty$, we get that

$$
\int_{\mathbb{R}^{N}} \nabla w \nabla \phi d x=0, \quad \forall \phi \in C_{0}^{\infty}\left(\mathbb{R}^{N}\right) .
$$

This is impossible, because $0 \neq w \in X$. If $\left\{y_{n}\right\}$ has a subsequence bounded in $\mathbb{R}^{N}$, up to a subsequence, it can be assumed that $y_{0}=\lim _{n \rightarrow \infty} y_{n}$, and sending $n \rightarrow \infty$ in (42), we get that

$$
\begin{aligned}
\int_{\mathbb{R}^{N}} \nabla w \nabla \phi d x-\int_{\mathbb{R}^{N}} V\left(x+y_{0}\right) w \phi d x & =0, \\
\forall \phi & \in C_{0}^{\infty}\left(\mathbb{R}^{N}\right) .
\end{aligned}
$$

It follows that

$$
\begin{aligned}
& \int_{\mathbb{R}^{N}} \nabla w\left(\cdot-y_{0}\right) \nabla \phi d x-\int_{\mathbb{R}^{N}} V(x) w\left(\cdot-y_{0}\right) \phi d x \\
& =0, \quad \forall \phi \in C_{0}^{\infty}\left(\mathbb{R}^{N}\right) .
\end{aligned}
$$

This implies that $w\left(\cdot-y_{0}\right)$ is a nonzero eigenfunction of $T$ corresponding to eigenvalue 0 . It contradicts Lemma 4.

Case 2. $\left\{w_{n}\right\}$ is vanishing; that is,

$$
\lim _{n \rightarrow \infty} \sup _{y \in \mathbb{R}^{N}} \int_{B_{\mathbb{R}^{N}}(y, 1)} w_{n}^{2} d x=0 .
$$

The Lions lemma (see, e.g., [6, Lemma 1.21]) shows that, for any $p \in(2,2 N /(N-2))$,

$$
\lim _{n \rightarrow \infty}\left\|w_{n}\right\|_{L^{p}}=0 \text {. }
$$

Here, $\|\cdot\|_{L^{p}}$ denotes $L^{p}\left(\mathbb{R}^{N}\right)$ norm. Equations (39) and (27) show that, as $n \rightarrow \infty$,

$$
\begin{aligned}
o(1)= & \left\langle\Phi^{\prime}\left(u_{n}\right), u_{n}\right\rangle \\
= & \int_{\mathbb{R}^{N}}\left|\nabla u_{n}\right|^{2} d x-\int_{\mathbb{R}^{N}} V(x) u_{n}^{2} d x \\
& +\int_{\mathbb{R}^{N}} f\left(u_{n}\right) u_{n} d x \\
= & \int_{\mathbb{R}^{N}}\left|\nabla u_{n}\right|^{2} d x+a_{0} \int_{\mathbb{R}^{N}} u_{n}^{2} d x-\int_{\mathbb{R}^{N}} V(x) u_{n}^{2} d x \\
& \quad-\int_{\mathbb{R}^{N}} g\left(u_{n}\right) u_{n} d x \\
= & \left\|u_{n}\right\|^{2}-\int_{\mathbb{R}^{N}} V(x) u_{n}^{2} d x-\int_{\mathbb{R}^{N}} g\left(u_{n}\right) u_{n} d x \\
= & \left\|u_{n}\right\|^{2}\left(1-\int_{\mathbb{R}^{N}} V(x) w_{n}^{2} d x-\int_{\mathbb{R}^{N}} \frac{g\left(u_{n}\right)}{u_{n}} w_{n}^{2} d x\right),
\end{aligned}
$$


where $g$ is defined in (15). It follows that

$$
\int_{\mathbb{R}^{N}} \frac{g\left(u_{n}\right)}{u_{n}} w_{n}^{2} d x+\int_{\mathbb{R}^{N}} V(x) w_{n}^{2} d x \longrightarrow 1,
$$

$$
n \longrightarrow \infty \text {. }
$$

By Lemma 6 and $w_{n} \rightarrow 0$ in $X$, we have

$$
\lim _{n \rightarrow \infty} \int_{\mathbb{R}^{N}} V(x) w_{n}^{2} d x=0 .
$$

Together with (49), this implies

$$
\int_{\mathbb{R}^{N}} \frac{g\left(u_{n}\right)}{u_{n}} w_{n}^{2} d x \longrightarrow 1, \quad n \longrightarrow \infty .
$$

By (39),

$$
\begin{aligned}
o(1)+c & =\Phi\left(u_{n}\right)-\frac{1}{2}\left\langle\Phi^{\prime}\left(u_{n}\right), u_{n}\right\rangle \\
& =\int_{\mathbb{R}^{N}} \widetilde{F}\left(u_{n}\right) d x
\end{aligned}
$$

Let

$$
E_{n}=\left\{x \in \mathbb{R}^{N}|| \frac{g\left(u_{n}(x)\right)}{u_{n}(x)} \mid \geq a_{0}-\delta\right\}
$$

By $\widetilde{F} \geq 0$, (17), and (52), the following is true:

$$
\begin{aligned}
\delta \operatorname{mes}\left(E_{n}\right) & \leq \int_{E_{n}} \widetilde{F}\left(u_{n}\right) d x \leq \int_{\mathbb{R}^{N}} \widetilde{F}\left(u_{n}\right) d x \\
& \leq c+o(1) .
\end{aligned}
$$

Here, $\operatorname{mes}(A)$ denotes the Lebesgue measure of $A$. It follows that

$$
\sup _{n} \operatorname{mes}\left(E_{n}\right)<+\infty \text {. }
$$

Let

$$
F_{n}=\left\{x \in \mathbb{R}^{N}|| \frac{g\left(u_{n}(x)\right)}{u_{n}(x)} \mid<a_{0}-\delta\right\} .
$$

Because $\delta \in\left(0, a_{0}\right)\left(\right.$ see $\left.\left(\mathbf{f}_{3}\right)\right)$, we have

$$
\begin{aligned}
\int_{F_{n}}\left|\frac{g\left(u_{n}\right)}{u_{n}} w_{n}^{2}\right| d x & \leq\left(a_{0}-\delta\right) \int_{\mathbb{R}^{N}} w_{n}^{2} d x \\
& \leq\left(a_{0}-\delta\right)\left\|w_{n}\right\|_{L^{2}}^{2} \leq \frac{a_{0}-\delta}{a_{0}}\left\|w_{n}\right\|^{2} \\
& =\frac{a_{0}-\delta}{a_{0}}<1 .
\end{aligned}
$$

Under the assumption $\left(\mathbf{f}_{\mathbf{1}}\right)$, there exists $C>0$ such that

$$
|f(t)| \leq C|t|, \quad \forall t \in \mathbb{R} .
$$

It follows that $\left|g\left(u_{n}\right)\right| \leq C\left|u_{n}\right|$ for some constant $C>0$. Then, by (55) and (47), for $2<p<2 N /(N-2)$,

$$
\begin{aligned}
\int_{E_{n}}\left|\frac{g\left(u_{n}\right)}{u_{n}} w_{n}^{2}\right| d x & \leq C \int_{E_{n}} w_{n}^{2} d x \\
& \leq C\left(\operatorname{mes}\left(E_{n}\right)\right)^{(p-2) / p}\left\|w_{n}\right\|_{L^{p}}^{2} \\
& \longrightarrow 0, \quad n \longrightarrow \infty .
\end{aligned}
$$

Combining (57) and (59) yields

$$
\begin{aligned}
& \underset{n \rightarrow \infty}{\limsup } \int_{\mathbb{R}^{N}}\left|\frac{g\left(u_{n}\right)}{u_{n}} w_{n}^{2}\right| d x \\
& \leq \limsup _{n \rightarrow \infty} \int_{F_{n}}\left|\frac{g\left(u_{n}\right)}{u_{n}} w_{n}^{2}\right| d x \\
& \quad+\limsup _{n \rightarrow \infty} \int_{E_{n}}\left|\frac{g\left(u_{n}\right)}{u_{n}} w_{n}^{2}\right| d x<1 .
\end{aligned}
$$

This contradicts (51).

From the two cases given above, it can be deduced that $\left\{u_{n}\right\}$ is bounded in $X$.

Lemma 8. The functional $\Phi$ satisfies the $(C)_{c}$ condition for every $c \in \mathbb{R}$.

Proof. Let $\left\{u_{n}\right\}$ be a $(C)_{c}$ sequence of $\Phi$. By Lemma $7,\left\{\left\|u_{n}\right\|\right\}$ is bounded. It follows that there exists $u \in X$ such that, up to a subsequence, $u_{n} \rightarrow u$ in $X$. Because the embedding $X \hookrightarrow$ $L_{\text {loc }}^{2}\left(\mathbb{R}^{N}\right)$ is compact, we get that, up to a subsequence,

$$
u_{n} \longrightarrow u \text { a.e. in } \mathbb{R}^{N} \text {. }
$$

As the proof of Lemma 6.15 of [6], we have $\Phi^{\prime}(u)=0$. It follows that

$$
\int_{\mathbb{R}^{N}}|\nabla u|^{2} d x+\int_{\mathbb{R}^{N}} f(u) u d x-\int_{\mathbb{R}^{N}} V(x) u^{2} d x=0 .
$$

We are going to prove that, up to a subsequence, $\| u_{n}-$ $u \| \rightarrow 0$ as $n \rightarrow \infty$.

By (39), as $n \rightarrow \infty$,

$$
\begin{aligned}
\left\langle\Phi^{\prime}\left(u_{n}\right), u_{n}\right\rangle= & \int_{\mathbb{R}^{N}}\left|\nabla u_{n}\right|^{2} d x+\int_{\mathbb{R}^{N}} f\left(u_{n}\right) u_{n} d x \\
& -\int_{\mathbb{R}^{N}} V(x) u_{n}^{2} d x=o(1) .
\end{aligned}
$$

By Lemma 6,

$$
\lim _{n \rightarrow \infty} \int_{\mathbb{R}^{N}} V(x) u_{n}^{2} d x=\int_{\mathbb{R}^{N}} V(x) u^{2} d x .
$$

Combining (62)-(64) yields

$$
\begin{gathered}
\lim _{n \rightarrow \infty}\left(\int_{\mathbb{R}^{N}}\left|\nabla u_{n}\right|^{2} d x+\int_{\mathbb{R}^{N}} f\left(u_{n}\right) u_{n} d x\right) \\
=\int_{\mathbb{R}^{N}}|\nabla u|^{2} d x+\int_{\mathbb{R}^{N}} f(u) u d x .
\end{gathered}
$$


Because $u_{n} \rightarrow u$ in $X$, by [17, Theorem 1.6], we have

$$
\liminf _{n \rightarrow \infty} \int_{\mathbb{R}^{N}}\left|\nabla u_{n}\right|^{2} d x \geq \int_{\mathbb{R}^{N}}|\nabla u|^{2} d x
$$

Because $t f(t) \geq 0$ for any $t \in \mathbb{R}$ (see (12)), by (61) and the Fatou lemma, we have

$$
\liminf _{n \rightarrow \infty} \int_{\mathbb{R}^{N}} f\left(u_{n}\right) u_{n} d x \geq \int_{\mathbb{R}^{N}} f(u) u d x
$$

Using (65)-(67), it can be deduced that, up to a subsequence,

$$
\begin{aligned}
& \lim _{n \rightarrow \infty} \int_{\mathbb{R}^{N}}\left|\nabla u_{n}\right|^{2} d x=\int_{\mathbb{R}^{N}}|\nabla u|^{2} d x, \\
& \lim _{n \rightarrow \infty} \int_{\mathbb{R}^{N}} f\left(u_{n}\right) u_{n} d x=\int_{\mathbb{R}^{N}} f(u) u d x .
\end{aligned}
$$

By (13), $\left\{D u_{n} f\left(u_{n}\right)-F\left(u_{n}\right)\right\}$ is a sequence of nonnegative functions. Because

$$
\begin{aligned}
& D u_{n} f\left(u_{n}\right)-F\left(u_{n}\right) \longrightarrow \operatorname{Duf}(u)-F(u) \\
& \text { a.e. in } \mathbb{R}^{N},
\end{aligned}
$$

by the Fatou lemma and (69), the following is true:

$$
\begin{aligned}
\int_{\mathbb{R}^{N}} & (D u(x) f(u(x))-F(u(x))) d x \\
\leq & \liminf _{n \rightarrow \infty} \int_{\mathbb{R}^{N}}\left(D u_{n}(x) f\left(u_{n}(x)\right)-F\left(u_{n}(x)\right)\right) d x \\
= & D \int_{\mathbb{R}^{N}} u(x) f(u(x)) d x \\
& -\limsup _{n \rightarrow \infty} \int_{\mathbb{R}^{N}} F\left(u_{n}(x)\right) d x .
\end{aligned}
$$

It follows that

$$
\int_{\mathbb{R}^{N}} F(u(x)) d x \geq \limsup _{n \rightarrow \infty} \int_{\mathbb{R}^{N}} F\left(u_{n}(x)\right) d x .
$$

By (12), we deduce that $F \geq 0$ in $\mathbb{R}$. Then, by

$$
F\left(u_{n}\right) \longrightarrow F(u), \text { a.e. in } \mathbb{R}^{N}
$$

and the Fatou lemma, the following holds:

$$
\int_{\mathbb{R}^{N}} F(u(x)) d x \leq \liminf _{n \rightarrow \infty} \int_{\mathbb{R}^{N}} F\left(u_{n}(x)\right) d x .
$$

Combining (72) and (74) leads to

$$
\lim _{n \rightarrow \infty} \int_{\mathbb{R}^{N}} F\left(u_{n}(x)\right)=\int_{\mathbb{R}^{N}} F(u(x)) d x
$$

By (10) and (11), it can be deduced that there exists $C>0$ such that

$$
|f(t)| \leq C|t|, \quad \forall \in \mathbb{R} .
$$

Then, by the mean value theorem, the Hölder inequality, and (76), we get that, for any $a, b \in \mathbb{R}$ and for any sufficiently small $\epsilon>0$, there exists $0<\theta<1$ such that

$$
\begin{aligned}
|F(a+b)-F(a)| & =|b f(a+\theta b)| \leq C|b| \cdot|a+\theta b| \\
& \leq C|a| \cdot|b|+C b^{2} \\
& \leq \frac{C \epsilon^{2}}{2} a^{2}+C\left(1+\frac{1}{2 \epsilon^{2}}\right) b^{2} .
\end{aligned}
$$

Because (61), (75), and (77) hold and $\left\{\left\|u_{n}\right\|\right\}$ is bounded, Theorem 2 of [18] shows that

$$
\lim _{n \rightarrow \infty} \int_{\mathbb{R}^{N}} F\left(u_{n}-u\right) d x=0 .
$$

It follows that, for every sufficiently small $\epsilon>0$,

$$
\lim _{n \rightarrow \infty} \int_{E_{n, \epsilon}} F\left(u_{n}-u\right) d x=0
$$

Here,

$$
E_{n, \epsilon}=\left\{x|| u_{n}(x)-u(x) \mid \leq \epsilon^{-1}\right\} .
$$

Equations (10) and (12) show that

$$
\begin{aligned}
& F(t)>0, \quad t \neq 0, \\
& \liminf _{|t| \rightarrow 0} \frac{F(t)}{t^{2}}>0 .
\end{aligned}
$$

Combining (81) and (82) yields that, for any sufficiently small $\epsilon>0$, there exists $C_{\epsilon}>0$ such that

$$
t^{2} \leq C_{\epsilon} F(t), \quad \forall t \in\left[-\epsilon^{-1}, \epsilon^{-1}\right] .
$$

By (79) and (83), we get that, for every sufficiently small $\epsilon>0$,

$$
\lim _{n \rightarrow \infty} \int_{E_{n, \epsilon}}\left|u_{n}-u\right|^{2} d x=0 .
$$

From

$$
\begin{aligned}
\epsilon^{-2} \operatorname{mes}\left(\mathbb{R}^{N} \backslash E_{n, \epsilon}\right) & \leq \int_{\mathbb{R}^{N} \backslash E_{n, \epsilon}}\left|u_{n}-u\right|^{2} d x \leq M \\
& :=\sup _{n} \int_{\mathbb{R}^{N}}\left|u_{n}-u\right|^{2} d x<\infty,
\end{aligned}
$$

we get that

$$
\operatorname{mes}\left(\mathbb{R}^{N} \backslash E_{n, \epsilon}\right) \leq M \epsilon^{2}, \quad n=1,2, \ldots
$$

By (86), the following holds:

$$
\begin{aligned}
& \int_{\mathbb{R}^{N} \backslash E_{n, \epsilon}}\left|u_{n}-u\right|^{2} d x \\
& \leq\left(\operatorname{mes}\left(\mathbb{R}^{N} \backslash E_{n, \epsilon}\right)\right)^{2 / N}\left(\int_{\mathbb{R}^{N}}\left|u_{n}-u\right|^{2^{*}} d x\right)^{2 / 2^{*}} \\
& \leq M^{\prime}\left(\operatorname{mes}\left(\mathbb{R}^{N} \backslash E_{n, \epsilon}\right)\right)^{2 / N} \leq M^{\prime} M^{2 / N} \epsilon^{4 / N},
\end{aligned}
$$


where $2^{*}=2 N /(N-2)$ and

$$
M^{\prime}=\sup _{n}\left(\int_{\mathbb{R}^{N}}\left|u_{n}-u\right|^{2^{*}} d x\right)^{2 / 2^{*}}<\infty .
$$

Combining (84) and (87) leads to

$$
\lim _{n \rightarrow \infty} \int_{\mathbb{R}^{N}}\left|u_{n}-u\right|^{2} d x=0
$$

Together with (68), this implies $u_{n} \rightarrow u$ in $X$. This completes the proof.

\section{Proof of Theorem 1}

For the proof of Theorem 1, the following abstract theorem, which is a corollary of [13, Theorem 2.4], is used.

Theorem 9. Let $E$ be a real Hilbert space with norm $\|\cdot\|$. Suppose that $J$ is a $C^{1}$-functional defined in $E$ and satisfies the following conditions:

$\left(\mathbf{A}_{1}\right) J$ satisfies the $(C)_{c}$ condition for every $c>0$, and $J(0) \geq$ 0 .

$\left(\mathbf{A}_{2}\right) J$ is an even functional; that is, $J(-u)=J(u)$ for every $u \in E$.

$\left(\mathbf{A}_{3}\right)$ There exist $c_{0}>J(0)$ and a closed subspace $Z$ of $E$ with finite codimension such that there exists $\rho>0$ such that

$$
\inf _{S_{\rho} \cap Z} J \geq c_{0}
$$

Here, $S_{\rho}=\{u \in E \mid\|u\|=\rho\}$.

$\left(\mathbf{A}_{4}\right)$ For any $n \in \mathbb{N}$, there exists a subspace $V \subset X$ with dimension larger than or equal to $n$ such that

$$
\sup _{V} J<+\infty
$$

Then, J has infinitely many pairs of critical points.

Proof. Arguing indirectly, assume that $J$ has only a finite number of critical points, say $n_{0}$. The codimension of $Z$ is here denoted by $m_{0}$. By $\left(\mathbf{A}_{\mathbf{4}}\right)$, there exists a subspace $V \subset Y$ with dimension larger than or equal to $n_{0}+m_{0}+1$ such that

$$
\sup _{V} J<+\infty
$$

This indicates that there exists $c_{\infty}>c_{0}$ such that

$$
\sup _{V} J<c_{\infty}
$$

Then, by [13, Theorem 2.4], $J$ has at least $\left(n_{0}+m_{0}+1\right)-m_{0}=$ $n_{0}+1$ critical points. This contradicts the assumption that $J$ has only $n_{0}$ critical points.

Let

$$
\lambda_{1}<\lambda_{2} \leq \lambda_{3} \leq \cdots \leq \lambda_{n} \leq \cdots
$$

be the eigenvalues of $T$ below the essential spectrum of $T$. Each $\lambda_{n}$ has been repeated in the sequence according to its finite multiplicity. Then, by Lemma 3, the following is true:

$$
\lim _{n \rightarrow \infty} \lambda_{n}=0, \quad \lambda_{n}<0, \forall n .
$$

Let

$$
n_{*}=\min \left\{n \in \mathbb{N} \mid a_{0}+\lambda_{n}>0\right\} .
$$

Here, $a_{0}$ is the constant in (10). From this definition,

$$
a_{0}+\lambda_{n_{*}}>0 \geq a_{0}+\lambda_{n_{*}-1}, \quad \text { if } n_{*}>1
$$

Let $\left\{E_{\lambda} \mid \lambda \in \mathbb{R}\right\}$ denote the spectral family of the operator $T$ defined by (3).

Let

$$
Z= \begin{cases}X, & \text { if } n_{*}=1 \\ \left(\mathrm{id}-E_{v}\right)\left(L^{2}\left(\mathbb{R}^{N}\right)\right) \cap X, & \text { if } n_{*}>1 .\end{cases}
$$

Here, id $: L^{2}\left(\mathbb{R}^{N}\right) \rightarrow L^{2}\left(\mathbb{R}^{N}\right)$ is the identity map and

$$
\nu=\frac{1}{2}\left(\lambda_{n_{*}}+\lambda_{n_{*}-1}\right) \text {. }
$$

It is easy to see that the codimension of $Z$ equals $n_{*}-1$.

Lemma 10. There exists $\varsigma>0$ such that

$$
\begin{aligned}
& \int_{\mathbb{R}^{N}}|\nabla u|^{2} d x+a_{0} \int_{\mathbb{R}^{N}} u^{2} d x-\int_{\mathbb{R}^{N}} V(x) u^{2} d x \\
& \geq \varsigma\|u\|^{2}, \quad \forall u \in Z .
\end{aligned}
$$

Proof. From (96) and (98), the following holds:

$$
\begin{aligned}
& \int_{\mathbb{R}^{N}}|\nabla u|^{2} d x+a_{0} \int_{\mathbb{R}^{N}} u^{2} d x-\int_{\mathbb{R}^{N}} V(x) u^{2} d x \\
& \geq \kappa \int_{\mathbb{R}^{N}} u^{2} d x, \quad \forall u \in Z .
\end{aligned}
$$

Here,

$$
\kappa=a_{0}+\lambda_{n_{*}}>0
$$

It follows that

$$
\begin{aligned}
\varsigma:= & \left\{\int_{\mathbb{R}^{N}}|\nabla u|^{2} d x+a_{0} \int_{\mathbb{R}^{N}} u^{2} d x\right. \\
& \left.-\int_{\mathbb{R}^{N}} V(x) u^{2} d x \mid u \in Z,\|u\|=1\right\} \geq 0 .
\end{aligned}
$$

If $\varsigma=0$, then there exists $\left\{u_{n}\right\} \subset Z$ satisfying $\left\|u_{n}\right\|=1$, $n=1,2, \ldots$, and

$$
\begin{gathered}
\lim _{n \rightarrow \infty}\left(\int_{\mathbb{R}^{N}}\left|\nabla u_{n}\right|^{2} d x+a_{0} \int_{\mathbb{R}^{N}} u_{n}^{2} d x\right. \\
\left.-\int_{\mathbb{R}^{N}} V(x) u_{n}^{2} d x\right)=0 .
\end{gathered}
$$


Up to a subsequence, it can be assumed that $u_{n} \rightarrow u_{0}$ in $X$ and $u_{n} \rightarrow u_{0}$ a.e. in $\mathbb{R}^{N}$. Since $Z$ is a closed subspace of $X$, by $u_{n} \rightarrow u_{0}$ in $X$, we have $u_{0} \in Z$. Because $u_{n} \rightarrow u_{0}$ in $X$, by [17, Theorem 1.6], we have

$$
\liminf _{n \rightarrow \infty} \int_{\mathbb{R}^{N}}\left|\nabla u_{n}\right|^{2} d x \geq \int_{\mathbb{R}^{N}}\left|\nabla u_{0}\right|^{2} d x
$$

Because $u_{n} \rightarrow u_{0}$ a.e. in $\mathbb{R}^{N}$, by the Fatou lemma, the following holds:

$$
\liminf _{n \rightarrow \infty} \int_{\mathbb{R}^{N}} u_{n}^{2} d x \geq \int_{\mathbb{R}^{N}} u_{0}^{2} d x
$$

Moreover, by Lemma 6,

$$
\lim _{n \rightarrow \infty} \int_{\mathbb{R}^{N}} V(x) u_{n}^{2} d x=\int_{\mathbb{R}^{N}} V(x) u_{0}^{2} d x .
$$

Combining (104)-(107) yields

$$
\int_{\mathbb{R}^{N}}\left|\nabla u_{0}\right|^{2} d x+a_{0} \int_{\mathbb{R}^{N}} u_{0}^{2} d x-\int_{\mathbb{R}^{N}} V(x) u_{0}^{2} d x \leq 0 .
$$

Because $u_{0} \in Z$, by (101) and (108), we have $u_{0}=0$. Then, by (104) and (107),

$$
\lim _{n \rightarrow \infty}\left(\int_{\mathbb{R}^{N}}\left|\nabla u_{n}\right|^{2} d x+a_{0} \int_{\mathbb{R}^{N}} u_{n}^{2} d x\right)=0 .
$$

This contradicts $\left\|u_{n}\right\|=1, n=1,2, \ldots$. Therefore, $\varsigma>0$.

Proof of Theorem 1. We are going to prove that the functional $\Phi$ satisfies the conditions $\left(\mathbf{A}_{\mathbf{1}}\right)-\left(\mathbf{A}_{\mathbf{4}}\right)$ in Theorem 9.

By Lemma 8, the functional $\Phi$ satisfies the condition $\left(\mathbf{A}_{\mathbf{1}}\right)$. By $\left(\mathbf{f}_{\mathbf{4}}\right), \Phi$ satisfies the condition $\left(\mathbf{A}_{2}\right)$.

By (10) and (11), it can be deduced that, for any $\epsilon>0$, there exists $C_{\epsilon}>0$ such that

$$
\left|F(t)-\frac{a_{0} t^{2}}{2}\right| \leq \epsilon t^{2}+C_{\epsilon}|t|^{\mu}, \quad \forall t \in \mathbb{R} .
$$

Here,

$$
\mu=\frac{2 N-2}{N-2} \in\left(2,2^{*}\right)
$$

Let $Z$ be the space defined by (98). It has a finite codimension. By Lemma 10 and (110), we have, for any $u \in Z$,

$$
\begin{aligned}
\Phi(u)= & \frac{1}{2} \int_{\mathbb{R}^{N}}|\nabla u|^{2} d x+\frac{a_{0}}{2} \int_{\mathbb{R}^{N}} u^{2} d x \\
& -\frac{1}{2} \int_{\mathbb{R}^{N}} V(x) u^{2} d x \\
& +\int_{\mathbb{R}^{N}}\left(F(u)-\frac{a_{0}}{2} u^{2}\right) d x \\
\geq & \frac{\varsigma}{2}\|u\|^{2}-\epsilon\|u\|_{L^{2}}^{2}-C_{\epsilon}\|u\|_{L^{\mu}}^{\mu} \\
\geq & \left(\frac{\varsigma}{2}-\epsilon\right)\|u\|^{2}-C_{\epsilon}\|u\|_{L^{\mu}}^{\mu} \\
\geq & \left(\frac{\varsigma}{2}-\epsilon\right)\|u\|^{2}-C^{\prime} C_{\epsilon}\|u\|^{\mu},
\end{aligned}
$$

where the positive constant $C^{\prime}$ comes from the Sobolev inequality $\|u\|_{L^{\mu}} \leq\left(C^{\prime}\right)^{1 / \mu}\|u\|, \forall u \in X$. Choosing $\epsilon=\varsigma / 4$ and $\rho=\left(\varsigma / 8 C^{\prime} C_{\epsilon}\right)^{1 /(\mu-2)}$, by (112), the following holds:

$$
\inf _{S_{\rho} \cap Z} \Phi \geq c_{0}:=\frac{\varsigma}{8} \rho^{2}>\Phi(0)
$$

It follows that $\Phi$ satisfies the assumption $\left(\mathbf{A}_{\mathbf{3}}\right)$.

Let $\vartheta \in C_{0}^{\infty}\left(\mathbb{R}^{N}\right)$ such that $\|\vartheta\|_{L^{2}}=1$ and supp $\vartheta \mathrm{C}$ $\left\{x \in \mathbb{R}^{N}|1<| x \mid<2\right\}$. Then, for the function $\vartheta_{\lambda}(x)=$ $\lambda^{-N / 2} \vartheta\left(\lambda^{-1} x\right),\left\|\vartheta_{\lambda}\right\|_{L^{2}}=1$ and supp $\vartheta_{\lambda} \subset\left\{x \in \mathbb{R}^{N}|\lambda<| x \mid<\right.$ $2 \lambda\}$. Then, by (8), it follows that, for $\lambda>R$,

$$
\begin{gathered}
-\int_{\mathbb{R}^{N}} V(x)\left|\vartheta_{\lambda}\right|^{2} d x+\int_{\mathbb{R}^{N}}\left|\nabla \vartheta_{\lambda}\right|^{2} d x \\
\leq-C \lambda^{-\beta}\|\vartheta\|_{L^{2}}^{2}+C \lambda^{-2}\|\nabla \vartheta\|_{L^{2}}^{2},
\end{gathered}
$$

where $C$ is a positive constant independent of $\lambda$. Because $\beta<$ 2 , it follows that there exists $\lambda_{0}>R$ such that

$$
\int_{\mathbb{R}^{N}}\left|\nabla \vartheta_{\lambda}\right|^{2} d x-\int_{\mathbb{R}^{N}} V(x)\left|\vartheta_{\lambda}\right|^{2} d x<0 \quad \text { for } \lambda \geq \lambda_{0} .
$$

Let $\psi_{n}=\vartheta_{2^{n} \lambda_{0}}, n \in \mathbb{N}$. These functions have mutually disjoint compact supports. Let

$$
V_{n}=\operatorname{span}\left\{\psi_{1}, \psi_{2}, \ldots, \psi_{n}\right\}
$$

Then, $V_{n}$ is an $n$-dimensional subspace of $X$. By (10) and (11), it can be deduced that, for any $\epsilon>0$, there exist $L_{\epsilon}>0$ and $K_{\epsilon}>0$ such that

$$
\begin{aligned}
& |F(t)| \leq \epsilon|t|^{2}, \quad \text { if }|t|>L_{\epsilon}, \\
& |F(t)| \leq K_{\epsilon}|t|^{3 / 2}, \quad \text { if }|t| \leq L_{\epsilon} .
\end{aligned}
$$

Because $\left\{\psi_{i} \mid i \in \mathbb{N}\right\}$ have mutually disjoint compact supports, by (115), we have, for $u=\sum_{i=1}^{n} t_{i} \psi_{i} \in V_{n}$,

$$
\Phi(u)=\frac{1}{2} \sum_{i=1}^{n} \alpha_{i} t_{i}^{2}+\int_{\mathbb{R}^{N}} F(u) d x
$$

where

$$
\begin{aligned}
\alpha_{i}=\int_{\mathbb{R}^{N}}\left|\nabla \psi_{i}\right|^{2} d x-\int_{\mathbb{R}^{N}} V(x)\left|\psi_{i}\right|^{2} d x & <0, \\
& i=1,2, \ldots, n
\end{aligned}
$$

Let

$$
\Omega_{n}=\bigcup_{i=1}^{n} \operatorname{supp} \psi_{i}
$$


Then, $\Omega_{n}$ is a bounded set in $\mathbb{R}^{N}$, since every $\psi_{i}$ has a compact support. By (117),

$$
\begin{aligned}
\int_{\mathbb{R}^{N}} F(u) d x= & \int_{\left\{x|| u(x) \mid>L_{\epsilon}\right\}} F(u) d x \\
& +\int_{\left\{x|| u(x) \mid \leq L_{\epsilon}\right\}} F(u) d x \\
\leq & \epsilon \int_{\mathbb{R}^{N}} u^{2} d x+K_{\epsilon} \int_{\Omega_{n}}|u|^{3 / 2} d x \\
\leq & \epsilon \sum_{i=1}^{n} \omega_{i} t_{i}^{2} \\
& +K_{\epsilon}\left(\operatorname{mes}\left(\Omega_{n}\right)\right)^{1 / 4}\left(\int_{\Omega_{n}} u^{2} d x\right)^{3 / 4} \\
= & \epsilon \sum_{i=1}^{n} \omega_{i} t_{i}^{2} \\
& +K_{\epsilon}\left(\operatorname{mes}\left(\Omega_{n}\right)\right)^{1 / 4}\left(\sum_{i=1}^{n} \omega_{i} t_{i}^{2}\right)^{3 / 4}
\end{aligned}
$$

where

$$
\omega_{i}=\int_{\mathbb{R}^{N}} \psi_{i}^{2} d x>0, \quad i=1, \ldots, n
$$

Choosing

$$
\epsilon=\frac{\min \left\{-\alpha_{i} \mid 1 \leq i \leq n\right\}}{4 \max \left\{\omega_{i} \mid 1 \leq i \leq n\right\}},
$$

by (118) and (121), we get that

$$
\Phi(u) \leq \frac{1}{4} \sum_{i=1}^{n} \alpha_{i} t_{i}^{2}+K_{\epsilon}\left(\operatorname{mes}\left(\Omega_{n}\right)\right)^{1 / 4}\left(\sum_{i=1}^{n} \omega_{i} t_{i}^{2}\right)^{3 / 4} .
$$

Since $\alpha_{i}<0$, for every $i$, (124) implies

$$
\sup _{V_{n}} \Phi<\infty
$$

Therefore, $\Phi$ satisfies the condition $\left(\mathbf{A}_{\mathbf{4}}\right)$ of Theorem 9.

Because $\Phi$ satisfies the conditions $\left(\mathbf{A}_{1}\right)-\left(\mathbf{A}_{4}\right)$ of Theorem 9, by Theorem 9 , it has infinitely many critical points. It follows that (6) has infinitely many solutions.

\section{Conflict of Interests}

The authors declare that there is no conflict of interests regarding the publication of this paper.

\section{Acknowledgments}

Shaowei Chen was supported by Science Foundation of Huaqiao University (13BS208) and Promotion Program for Young and Middle-Aged Teacher in Science and Technology Research of Huaqiao University (ZQN-PY119).

\section{References}

[1] P. D. Hislop and I. M. Sigal, Introduction to Spectral Theory. With Applications to Schrödinger Operators, vol. 113 of Applied Mathematical Sciences, Springer, New York, NY, USA, 1996.

[2] J. Weidmann, Linear Operators in Hilbert Spaces, vol. 68 of Graduate Texts in Mathematics, Springer, Berlin, Germany, 1980.

[3] M. F. Furtado, L. A. Maia, and E. A. Silva, "On a double resonant problem in $\mathbb{R}^{N}$," Differential and Integral Equations, vol. 15, no. 11, pp. 1335-1344, 2002.

[4] Z. Liu, J. Su, and Z.-Q. Wang, "Elliptic systems on $\mathbb{R}^{N}$ with nonlinearities of linear growth," in Progress in Variational Methods, vol. 7 of Nankai Series in Pure, Applied Mathematics and Theoretical Physics, pp. 90-106, World Scientific, Hackensack, NJ, USA, 2011.

[5] Z. Liu and Z.-Q. Wang, "Existence of a positive solution of an elliptic equation on $\mathbb{R}^{N}$," Proceedings of the Royal Society of Edinburgh, Section A, vol. 134, no. 1, pp. 191-200, 2004.

[6] M. Willem, Minimax Theorems (Progress in Nonlinear Differential Equations and Their Applications), vol. 24, Birkhäuser, Boston, Mass, USA, 1996.

[7] N. P. Các, "On an elliptic boundary value problem at double resonance," Journal of Mathematical Analysis and Applications, vol. 132, no. 2, pp. 473-483, 1988.

[8] K.-C. Chang, Infinite-Dimensional Morse Theory and Multiple Solution Problems, vol. 6 of Progress in Nonlinear Differential Equations and their Applications, Birkhäuser, Boston, Mass, USA, 1993.

[9] N. Hirano, S. Li, and Z.-Q. Wang, "Morse theory without (PS) condition at isolated values and strong resonance problems," Calculus of Variations and Partial Differential Equations, vol. 10, no. 3, pp. 223-247, 2000.

[10] E. Landesman and A. Lazer, "Nonlinear perturbations of linear elliptic boundary value problems at resonance," Journal of Applied Mathematics and Mechanics, vol. 19, pp. 609-623, 1970.

[11] S. Chen and S. Li, "Splitting lemma at infinity and a strongly resonant problem with periodic nonlinearity," Calculus of Variations and Partial Differential Equations, vol. 27, no. 1, pp. 105123, 2006.

[12] Z. Shen and S. Liu, "On asymptotically linear elliptic equations in $\mathbb{R}^{N}$," Journal of Mathematical Analysis and Applications, vol. 392, no. 1, pp. 83-88, 2012.

[13] P. Bartolo, V. Benci, and D. Fortunato, "Abstract critical point theorems and applications to some nonlinear problems with 'strong' resonance at infinity,' Nonlinear Analysis. Theory, Methods \& Applications, vol. 7, no. 9, pp. 981-1012, 1983.

[14] M. Reed and B. Simon, Methods of Modern Mathematical Physics. IV. Analysis of Operators, Academic Press, New York, NY, USA, 1978.

[15] J. Weidmann, "The virial theorem and its application to the spectral theory of Schrödinger operators," Bulletin of the American Mathematical Society, vol. 73, pp. 452-456, 1967. 
[16] G. Cerami, "An existence criterion for the critical points on unbounded manifolds," Istituto Lombardo, Accademia di Scienze e Lettere, Rendiconti A, vol. 112, no. 2, pp. 332-336, 1978 (Italian).

[17] M. Struwe, Variational Methods, Springer, Berlin, Germany, 1996.

[18] H. Brézis and E. Lieb, "A relation between pointwise convergence of functions and convergence of functionals," Proceedings of the American Mathematical Society, vol. 88, no. 3, pp. 486490, 1983. 


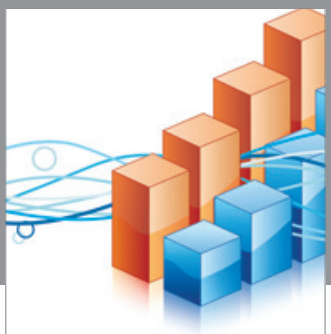

Advances in

Operations Research

vatem alat4

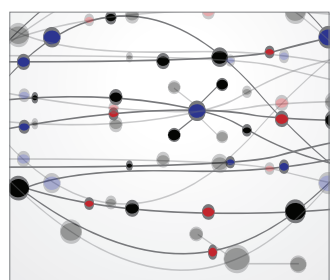

\section{The Scientific} World Journal
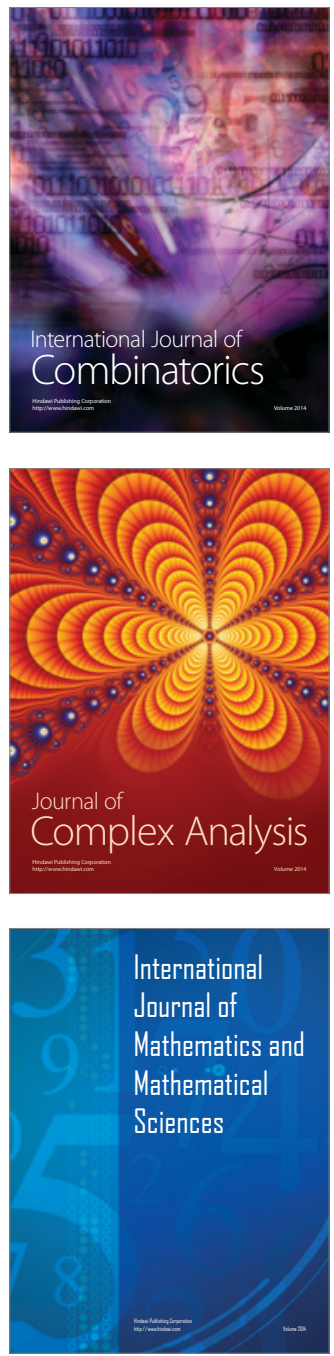
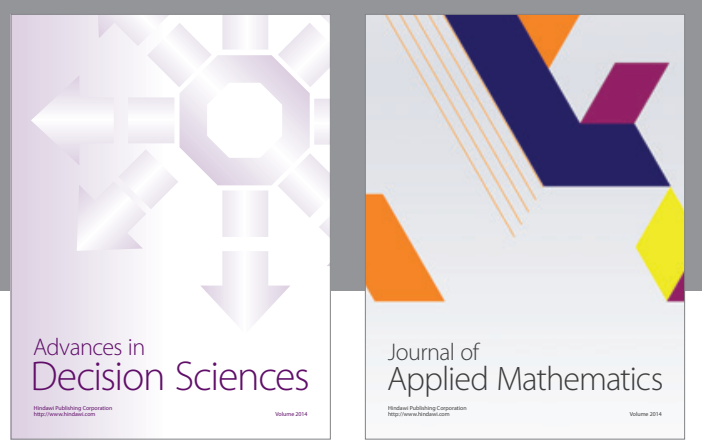

Algebra

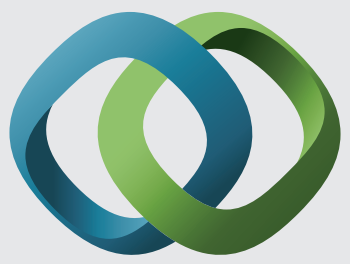

\section{Hindawi}

Submit your manuscripts at

http://www.hindawi.com
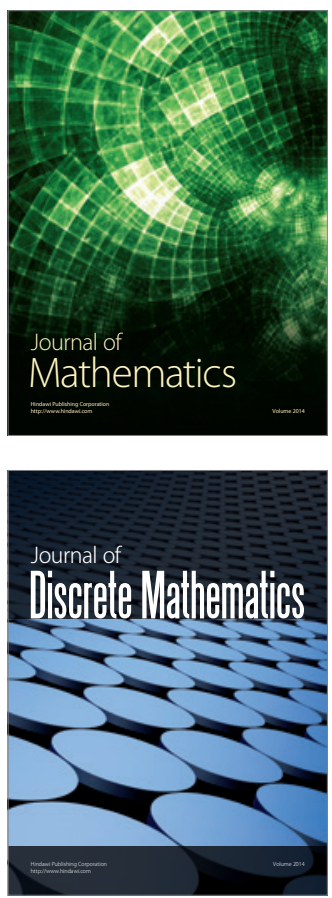

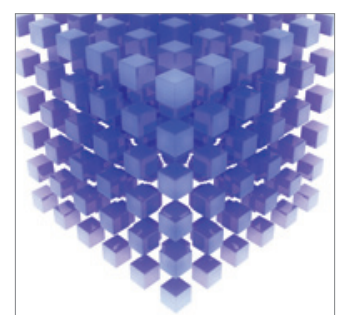

Mathematical Problems in Engineering
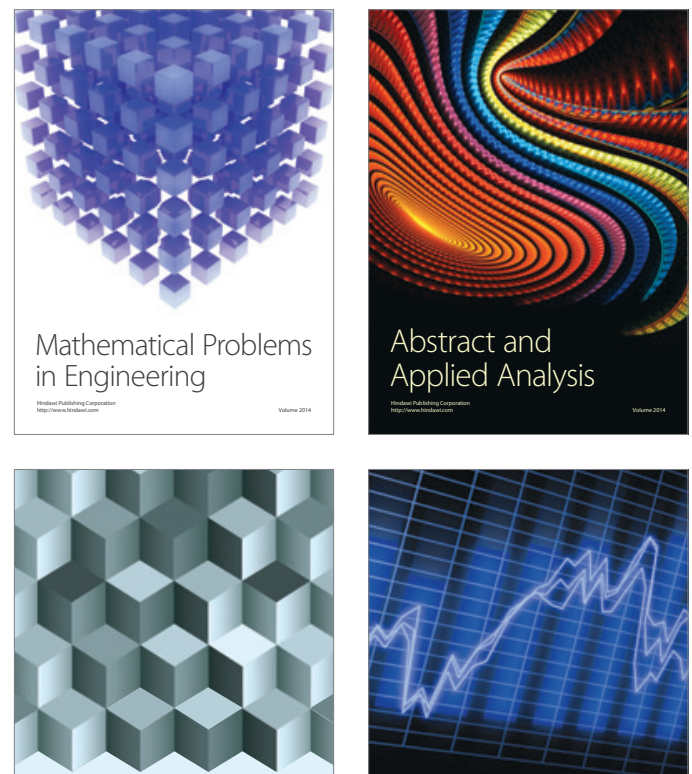

Journal of

Function Spaces

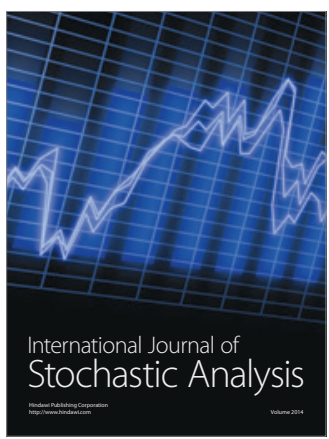

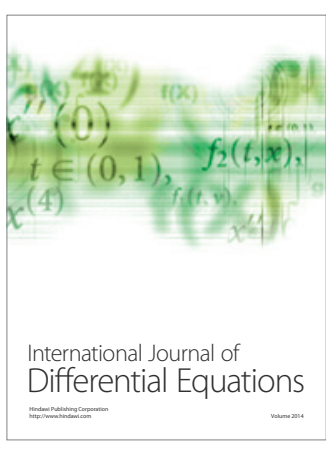
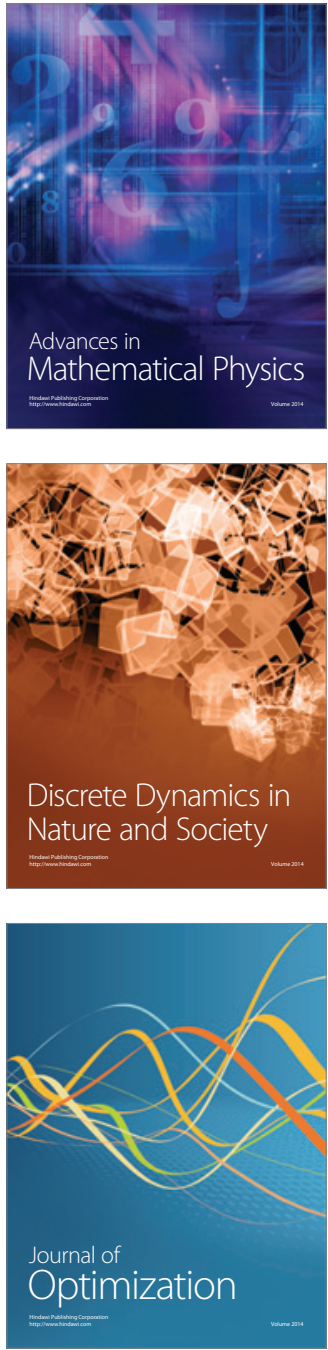